\title{
CONSIDERACIONES ACERCA DE LA SUSTANCIA Y LA IDENTIDAD PERSONAL EN LOCKE
}

En tres artículos anteriores, publicados en este mismo anuario, ${ }^{1}$ he sostenido, a propósito de Descartes, la tesis de que la filosofía de los racionalistas y los empiristas se ve infectada del principio cartesiano de la certeza o principio epistemológico fundamental $(P E F) .^{2}$

Descartes usa el $P E F$ para justificar su dualismo metafísico y esto plantea una dificultad general, a saber: si las personas son en realidad —es decir, más allá del estado encarnado que disfrutan en este mundo- egos o sustancias espirituales. ¿Cómo fijar las condiciones de su identidad?

Esto es, si las personas no son esencialmente seres encarnados - a pesar de las apariencias en contrario-, sino egos cartesianos - y a éstos se les clasifica como sustancias inmateriales-, entonces será necesario ofrecer las condiciones de identidad para estas sustancias, pues se han abrogado las condiciones de identidad normales (o aparentes) para egos encarnados.

Parece entonces que nos encontramos ante una tarea esencialmente filosófica: la tarea metafísica de fijar las condiciones de identidad para continuantes, como son o parecen ser las sustancias inmateriales.

Descartes no dice nada a este respecto y su silencio puede interpretarse por lo menos en dos sentidos: o bien pensó que no había dificultad, pues la sustancia inmaterial que es el ego se muestra a cada conciencia en forma inmediata y continua y esta experiencia es todo lo que se puede decir al respecto, es decir, aparte de esta experiencia inmediata de la sustancia inmaterial no hay otra cosa para la cual podamos postular condiciones de identidad en forma independiente; o bien, se percató del problema y decidió callar, por razones aparentemente obvias.

En contraste con Descartes, Locke es explícito y abundante a este respecto. Esta actitud de Locke muestra que reconoce la existencia del problema y decide ofrecer una solución al mismo.

Mi interpretación de Locke asume, por un lado, que Locke es dualista, y por el otro, que acepta el PEF. Dados estos dos compromisos, la solución de

1 Cf. "Acerca de la tesis filosófica del pensamiento en relación con la tesis del lenguaje de R. Descartes" (Diánoia, 1976), "EI realismo sustancial de Renato Descartes" (ibid., I977), "El principio de la certeza en Descartes" (ibid., 1978).

2 Sobre el PEF véase mi artículo en Diánoia, 1978. La idea general que subyace dicho principio es que los datos inmediatos de la propia conciencia son los que constituyen el fundamento de los objetos materiales y las personas. 
Locke debe ofrecer un criterio de identidad para las personas que sea inmaterial y que, por otra parte, cumpla con los requisitos del PEF. Estos compromisos son los que llevan a Locke a aceptar la memoria-conciencia como el criterio de identidad de las personas. Sin embargo, ambos compromisos no agotan ni determinan la solución de Locke.

Ahora bien, esta interpretación debe satisfacer, por un lado, la teoría positiva de Locke acerca de lo que es una sustancia, y por otro, su tesis de que las personas no son sustancias. Mi tesis a este respecto consiste en afirmar que, aun cuando Locke tiene cosas muy interesantes que decir acerca de las sustancias, hay en su argumento un non-sequitur que va desde el carácter que atribuye a la sustancia hasta su tesis acerca del tipo de identidad del continuante que es una persona. ${ }^{3}$ Estas consideraciones pueden alimentar algunas dudas importantes que conectan la discusión de Locke con la perspectiva contemporánea de los problemas de metafísica.

Comenzaré haciendo algunas consideraciones centrales acerca de la teoría de la sustancia de Locke, para después examinar la tesis de que las personas no son sustancias y, finalmente, plantear algunas interrogantes a la posición de Locke.

\section{LA SUSTANCIA}

De la sustancia dice Locke, en su Ensayo sobre el entendimiento humano: *

La idea que tenemos entonces, a la cual damos el nombre general de sustancia, no es otra sino el supuesto, pero desconocido, soporte de esas cualidades que encontramos que existen y que, imaginamos, no pueden substituir sine re substance, sin algo que les dé soporte; a este soporte lo llamamos sustancia; lo cual, de acuerdo con la verdadera significación de la palabra, en lenguaje común, es estar debajo de... o sostener. (II, XXIII, 2.)

De la cita anterior se desprende que Locke plantea la cuestión filosófica de la sustancia en términos de cualidades y propiedades y de un sujeto concebido como un soporte que sustenta las cualidades. El sujeto debe concebirse como un soporte de las cualidades; es decir, porque las cualidades o propiedades están unidas en un soporte, existe el individuo o continuante.

Locke introduce inmediatamente un problema acerca de ese sujeto o so-

3 Es decir, la tesis de la sustancia de Locke introduce una distinción apariencia-realidad que permite distinguir entre la ruta de un continuante y nuestra impresión de esa ruta. $\mathrm{Ni}$ de la consideración de la naturaleza de las sustancias ni de las consideraciones acerca de los predicados mentales se sigue que una persona no sea un continuante clasificable como una sustancia. "Continuante" es un sustantivo que debe leerse en una forma neutral, pues sólo pretende introducir la noción de cosa que persiste; luego vendrán las teorías que explican en qué consiste la identidad o unidad de un continuante.

- Utilizo la edición de A. C. Frazer publicada por Dover. Las traducciones son mías. 
porte, a saber, que mientras no tenemos dificultad (de principio) en conocer esas cualidades o propiedades, sí la tenemos para conocer qué es ese soporte, esto es, en qué consiste, cómo sostiene esas cualidades o propiedades.

Mientras más de esas cualidades coexistentes unimos en una idea compleja, bajo un nombre, más precisa y determinada hacemos la significación de esa palabra; sin embargo, no se la hace por ello más capaz de certeza universal, respecto de otras cualidades no contenidas en nuestra idea compleja, ya que no percibimos la conexión o dependencia de unas con otras, pues ignoramos, a la vez, la constitución real en la que están fundadas, y cómo surgen de ella. (IV, VI, 10.)

La cuestión de la sustancia se puede ver también — según Locke- como la cuestión de la unidad de las diferentes cualidades bajo un mismo nombre. Podemos así unir, bajo el nombre "oro", las cualidades de metal, amarillo, maleable, etc., pero no sabemos cómo de su esencia real resultan esas cualidades, ni cómo esa esencia las mantiene unidas y, por lo tanto, evita que se separen. Pero tampoco podemos saber que el oro no comprenda un número mayor de esas cualidades; no sabemos si al número $n$ de cualidades habría que agregar una cualidad más

Los principios de unidad que son las sustancias o sustratos nos son desconocidos en este sentido fundamental y esto produce un escepticismo epistemológico con respecto a la determinación de la sustancialidad de un ente dado. Suponemos un sustrato, pero no sabemos cuál es su constitución ni cómo unifica sus elementos.

Sin embargo, no es claro aún en dónde se localiza el escepticismo epistemológico respecto de las sustancias. Una posibilidad extrema consiste en pensar que el sustrato o sostén existe y que no podemos conocerlo. Es decir, el sustrato estaría más allá de las propiedades - las cuales, en nuestro ejemplo del oro, serían el amarillo, la maleabilidad, el peso $x$, etc.- y habría un velo que hace imposible conocerlo. A este respecto surge un pregunta: el conocimiento de ese sustrato o esencia real ¿seria, si fuera posible, similar al conocimiento que tenemos de las propiedades del oro? Si así fuera, quizás estaríamos en mejores condiciones de determinar por qué es imposible obtenerlo y qué tipo de imposibilidad es ésta. De no ser éste el caso, resultaría muy diff́cil entender la tesis de Locke.

Otra interpretación consiste en pensar ese sustrato, no como una propiedad más, sino como la conexión o relación legal entre las propiedades, misma que explicaría por qué el oro es amarillo, maleable, etc. Según esta interpretación, el sustrato o esencia real no necesitaría concebirse como un ente observable, y la dificultad epistemológica no consistiría en trascender un velo epistemológico postulado a priori, sino en tener conocimiento de las pro- 
piedades que componen una sustancia y de las relaciones de origen, conexión, precedencia temporal, etc., que se dan entre esas propiedades.

Locke se expresa, a través del Ensayo, en una forma por lo menos ambigua. En apoyo de la segunda interpretación antes enunciada dice:

¿Qué debemos hacer para mejorar nuestro conocimiento de los seres sustanciales? ...La experiencia nos debe enseñar aqui lo que la razón no puede enseñarnos: y solamente con dificultad puedo conocer con certeza cuáles otras cualidades coexisten con las de mi idea compleja... (IV, XII, 9.)

Según esto, la dificultad es empírica, contingente, y tenemos la capacidad de resolverla y lograr auténtica certeza en el conocimiento de los sustratos o esencias reales. Esta es la inversión de la tesis de Descartes, a saber, el mismo PEF, el mismo intuicionismo, pero ahora sobre un principio empirista, y la seguridad de que podemos alcanzar la certeza, sólo que gradualmente.

Según Locke, la experiencia es la que debe revelarnos la determinación del complejo de propiedades que componen una entidad como el oro. La idea de Locke es que, al conocer las otras propiedades del oro -aquellas que no conocemos aún-, o al conocer mejor sus propiedades establecidas, puede llegar a determinarse su composición y puede explicarse por qué nos aparece como maleable, amarillo, etc.

No quiero afirmar que la cuestión a la que se dirigen los metafísicos, conocida como la cuestión de la unidad o identidad de las sustancias, sea la cuestión que Locke considera. Es más pertinente decir que Locke interpretó la cuestión metafísica de la identidad como su propia cuestión de la unidad, y ésta como el descubrimiento empírico de las propiedades y su coexistencia y de cómo y por qué esos conjuntos de propiedades coexisten y forman clases naturales como el oro.

Para Locke, el descubrimiento empírico de las clases naturales explica por qué algo es una sustancia y por qué es legítimo aplicarle un término general o sortal en diferentes ocasiones. Un continuante como el oro es una sustancia porque es una clase natural, y es una clase natural porque, en él, $n$ propiedades coexisten de una manera determinada y se originan en cierta forma, estableciendo mutuas relaciones de conexión, dependencia, prioridad, etc., y esta constitución y continuidad legitima nuestro uso del sortal "oro".

Resumamos entonces las notas sobresalientes de la teoría de la sustancia de Locke:

Locke tiene una concepción positiva, y otra negativa, de la sustancia. De acuerdo con esta última, las sustancias son compuestos que determinan a priori la continuidad de un individuo, sin que se explique cómo ocurre esto.

La concepción positiva introduce la idea de un compuesto atómico que 
constituye la esencia real de un continuante individual frente a su esencia aparente o nominal. La esencia real o constitución interna está regida por leyes que originan causalmente las propiedades, cualidades o fuerzas de las cosas, las cuales a su vez causan en nosotros las ideas o el conocimiento de las mismas. Las leyes naturales rigen los individuos o continuantes y los agrupan en clases naturales. El continuante es un individuo en virtud de la ley natural que lo gobierna y tiene importancia y existe porque junto con otros forma una clase natural.

El sustrato o constitución interna o esencia real no lo percibimos y, por tanto, no lo conocemos, piensa Locke; podemos llegar a conocerlo, pero ese conocimiento tendrá que ser empírico. Las propiedades, cualidades o fuerzas las conocemos parcialmente. Hay, pues, una relatividad epistemológica combinada con un realismo esencialista $o$ absoluto de la sustancia. ${ }^{4}$ Los individuos son algo determinado y fijo más allá de cómo nos aparecen y de la idea que tenemos de ellos. Podemos llegar a conocerlos, es decir, podemos llegar a conocer lo que realmente son, trascendiendo la apariencia que tienen, mas para llegar a trascender la apariencia tenemos que basarnos en la experiencia. ${ }^{5}$

\section{LA IDENTIDAD DE LAS PERSONAS}

Locke ofrece una doble teoría para dar cuenta de la identidad de lo que comúnmente denominamos seres humanos. Para él hay que distinguir, bajo esa denominación ordinaria, "hombre" por un lado y "persona" por el otro. Ambos designan continuantes y tienen una identidad, pero Locke se rehúsa a categorizar esa identidad bajo el concepto de sustancia y, por tanto, rehúsa aplicarles su interesante teoría acerca de la sustancia. ${ }^{6}$

4 No sólo no se trata de tesis verificacionistas, sino de una tesis plenamente realista, según lo observaron Leibniz en su tiempo _cf. Nouveaux Essais, III, VI y X- y $\mathbf{H}$. Putman en nuestros días: cf. "The Meaning of 'Meaning' " en Mind, Language, and Reality: Philosophical Papers, vol. 2, Cambridge University Press, 1975. (Una reseña de este libro aparece en Critica, vol. VII, núm. 36, diciembre de 1980.)

5 Para una discusión más amplia de la teoría de Locke de la sustancia pueden consultarse: J. L. Mackie, Problems from Locke, Oxford University Press, 1976, y el artículo de Martha Brand Bolton "Substances, Substrata and Names of Substances in Locke's Essay", Philosophical Review, octubre de 1976.

- Rechazar que las personas sean sustancias implica negar que tengan una esencia real diferente de su esencia nominal y que por lo tanto, paulatina y empíricamente se vaya descubriendo su naturaleza, esto es, las leyes que rigen su composición y que determinan las clases naturales a las que pertenecen.

No obstante, las personas son continuantes y tienen identidad. ¿Se trata entonces de entidades con una identidad flotante, cuasi ficticias vis $\grave{a}$ vis un universo de sustancias individuales agrupadas en clases naturales? $L a$ idea es que las personas son individuos, continuantes; forman clases naturales pero no hay en ellas un principio unificador que sea un sustrato o esencia real. Locke no niega que las personas formen una clase, aunque tampoco justifica esa tesis. Por otra parte, si las personas no forman clases naturales $y$, por 
Para Locke, "hombre" designa el concepto de una sustancia material y un ego o yo. Sin embargo, el compuesto que así resulta no es una sustancia. Acerca de "mismo animal" y "misma persona" dice Locke:

Digo que en todos aquellos casos en que nuestra conciencia se interrumpe y perdemos de vista nuestros pasados yos, surgen dudas acerca de si somos la misma cosa pensante, esto es, si somos la misma sustancia o no. Las cuales dudas, razonables o no, en nada tocan la identidad personal. La cuestión de la identidad personal es acerca de lo que constituye la misma persona y no acerca de si se trata de la misma idéntica sustancia, la cual siempre piensa en la misma persona, todo lo cual nada importa en este caso. Diferentes sustancias quedan unidas en una persona mediante la misma conciencia - cuando participan de ella- así como diferentes cuerpos quedan unidos en un animal porque constituyen la misma vida, cuya identidad queda preservada a través de ese cambio de sustancias por la unidad de una vida continua. (II, XXVII, 10 ; p. 450 .)

De acuerdo con esto, la mismidad de vida continua nos da mismidad de animal, sin importar los cuerpos o sustancias en las que el animal resida.

Esta manera de hablar pone de manifiesto una diferencia importante con respecto a la manera de hablar de la identidad de una sustancia física o material, a saber, que la identidad de la persona no está en el mundo para que se la descubra. Por el contrario, la identidad de una persona va constituyéndose o, por así decir, autoconstruyéndose. Cuando trazamos la identidad de una persona perseguimos la continuidad de esa conciencia-memoria y, a través de esa búsqueda, logramos encadenar o unificar diferentes sustancias, hechos, estados, etc., donde ha residido esa misma conciencia-memoria que forma o constituye, así, la misma persona. En cambio, el mismo hombre resulta del mismo cuerpo, con una figura dada, y la misma sustancia espiritual, ya tenga la misma conciencia o no. (Ibid., 15.)

Sin embargo, es obvio que la conciencia, tanto cuanto pueda extenderse -incluso a edades pasadas-, une en la misma persona existencias y acciones muy remotas en el tiempo, al igual que las existencias y acciones del momento inmediatamente precedente, de manera que, sea lo que sea lo que tenga la conciencia de acciones presentes y pasadas, es la misma persona a la cual todas ellas pertenecen. (II, XXVII, 16.)

La conciencia_es, entonces, la que permite constituir o construir la mismidad

lo tanto, no son sustancias, ¿'se seguiria de esto que Locke desea garantizar en su teoria la singularidad irremplazable $e$ insustituible de las personas? No encuentro en sus textos nada que vaya en este sentido. 
de los continuantes que son las personas. "Conciencia" es un sortal que nos permite unir acciones, recuerdos, hechos, etc., y formar así una misma persona. Según Locke, "conciencia" es, a la vez, una condición necesaria y suficiente de "persona", es decir, "persona" puede analizarse o descomponerse en términos de estadios de conciencia:

Puesto que la conciencia siempre acompaña al pensamiento y esto es lo que hace a cada uno lo que se llama yo, ${ }^{7}$ y por tanto lo distingue de otros seres pensantes, en esto solamente consiste la identidad personal, i. e., la mismidad de un ser racional, y tanto como esta conciencia pueda extenderse a cualquier acción pasada o pensamiento, hasta allá se extiende la identidad de esa persona. (Ibid., 11.)

Lo que Locke no nos dice - según veremos- es cómo se lleva a cabo esa construcción de "persona" a partir de los estadios de conciencia."

Ahora, si bien es cierto que - según Locke- la identidad de la persona no es la identidad ni de una sustancia espiritual ni de una sustancia material, esto no quiere decir que la conciencia continua de un individuo no tenga nada que ver con la sustancia. Antes al contrario:

Estoy de acuerdo en que la opinión más probable es que esta conciencia está anexada a, y es la cualidad de, una sustancia inmaterial espiritual. (II, XXVII, 25.)

Más aún; parece que, por una parte, en este mundo la conciencia necesita residir en una sustancia, aun cuando no sea siempre la misma, y esa sustancia espiritual estará siempre anexada a otra sustancia material - la cual tampoco tiene que ser la misma- para formar al hombre. (Véase sobre esto II, XXVII, 25.)

Por otra parte, Locke piensa, sin embargo, que la unión de la conciencia con la sustancia espiritual y con la sustancia material es solamente contingente y que una misma conciencia puede transmigrar de una sustancia a otras indefinidamente y en esta transmigración, que bien puede ser anárquica, siempre va uniendo existencias y construyendo una misma persona o ego.

En este sentido, la teoría de Locke posee un carácter extremo, pues en la

7 El yo o ego se constituye o hace por el pensamiento; sin él no existirfa, y no habría racionalidad ni responsabilidad. Dicho en otra forma, no hay una esencia real por aprehender, sino un ego que se crea por el pensamiento reflexivo.

B Es decir, Locke no fija el alcance de "se puede exceder" y de "conciencia", dando lugar a interpretaciones que van desde lo absurdo hasta lo vacuo - según ha expuesto A. Flew en su articulo "Locke and the Problem of Personal Identity", Philosophy, 1951. Esta carencia es un síntoma de otra más grave, a saber, Locke no expone el mecanismo mediante el cual vamos a unificar esas conciencias en un mismo y único ego diferente a otros egos. Los autores neolockeanos citados en la nota 20 han intentado remediar esto. 
construcción de la identidad o unidad de una persona no intervienen la sustancia espiritual ni la sustancia material ni ninguna otra. Según quedó asentado anteriormente, la conciencia debe ser capaz por sí misma de ofrecer un análisis completo de la identidad de la persona. ( $C f$. II, XXVII, 25.)

Así, pues, Locke no está seguro de que las personas no sean sustancias espirituales según las concibió Descartes, es decir, sustancias cuyo atributo esencial es la conciencia. Pero la consideración de algunos casos posibles o contrafácticos ${ }^{10}$ lo lleva a negar que las personas puedan ser sustancias espirituales.

Son tres las razones que llevan a Locke a negar que las personas sean sustancias $y$, por lo tanto, a ofrecer una explicación diferente de su identidad: en primer lugar, las dificultades que encuentra con los casos posibles de Néstor y Tersistes, del príncipe y el mendigo; en segundo lugar, las referentes a la atribución de culpa y mérito $y$, en tercer lugar, su preferencia por una teoría que asegure la transparencia epistemológica de las personas.

Respecto al primer tipo de razón, Locke elimina la teoría de la sustancia como apta para capturar la identidad de las personas imaginando varias situaciones. Por ejemplo: hay un príncipe y un mendigo. Si cambiamos el alma del príncipe al mendigo, no tendremos ni la misma persona ni el mismo hombre. No tendremos el mismo hombre porque los cuerpos serán diferentes, y no tendremos la misma persona porque los recuerdos y la conciencia del príncipe no se habrán mudado al cuerpo del mendigo junto con su alma.

Todo el argumento anterior descansa en la premisa de Locke de que "misma alma" $\neq$ "misma conciencia". Esta premisa supone una peculiar teoría de la sustancia espiritual o inmaterial, de acuerdo con la cual el compuesto -atómico - que constituye la sustancia espiritual solamente mantiene una relación contingente con las propiedades o atributos mentales como la conciencia, la memoria, etc. A su vez, la sustancia espiritual mantiene relaciones meramente contingentes con las sustancias materiales o corporales en las que pueda residir.

El argumento para eliminar la identidad "persona = sustancia material"

$9 \mathrm{Si}$ las sustancias tienen atributos esenciales en el sentido de Descartes, no habría posibilidad de distinguir sustancia de conciencia y Locke no podria introducir sus casos contrafácticos. Sin embargo, podría aducir que la noción de sustancia (pensante) de Descartes es incoherente. Véanse las descripciones de casos de misma conciencia y diferente sustancia en $i b i d ., 21$ y 25.

10 Sobre el valor de la filosofía que utiliza casos contrafácticos o de ciencia-ficción concuerdo con lo que dice Geach en "Ontological Relativity and Relative Identity": "A pesar de enfrentar casos realmente extraños, la lógica de la identidad y de los nombres propios es demasiado central a nuestro esquema conceptual como para que se revise así sea ligera. mente; no la vamos a revisar solamente por conceder que un caso de ciencia-ficción describe un posible estado de cosas." En W. Munitz (ed.), Logic \& Ontology, New York University Press, 1973. 
consiste en observar que la persona permanece la misma aun cuando el cuerpo sufra cambios físicos y químicos. Locke se conforma con comprobar este hecho del mundo sin preguntarse si, más allá de las mutaciones del cuerpo, no podríamos descubrir la identidad de la persona con algo material. ${ }^{11}$ Locke no insiste en la defensa de su argumento porque los casos contrafácticos lo han convencido de que la persona/conciencia puede transmigrar de un cuerpo a otro.

¿Por qué concede Locke tanto peso a los casos contrafácticos? ¿O acaso, por el contrario, los casos contrafácticos le sirven solamente de pretexto para tomar partido por la conciencia como condición necesaria y suiciente de la personalidad? Quizás haya convicciones cartesianas detrás de esa elección. ${ }^{12}$ Haré dos tipos de consideraciones al respecto.

Un primer punto concierne lo completo de las descripciones de los casos posibles. Otro concierne la capacidad del criterio o condición o principio de identidad de las personas de Locke frente a los casos problema y los casos reales.

Respecto de la descripción de esos casos, cabe abrigar dudas acerca de si entendemos lo que sería que Néstor y Tersites pudieran intercambiar conciencias. ${ }^{13}$ Locke parece pensar que la conciencia es como una nariz o una oreja que se puede pasar o trasplantar de un cuerpo a otro. Sin embargo, "pasar" o "trasplantar" la conciencia de un cuerpo a otro, o de una sustancia espiritual a otra, es algo que no parece estar bien definido. Parte de la dificultad a la que debe enfrentarse la descripción de estos casos consiste en la oscuridad del concepto mismo de conciencia y de otros conceptos afines. Sobre esto volveré al examinar la segunda dificultad de Locke.

Pero entonces, si la descripción de los casos contrafácticos no es satisfactoria, ęcómo podemos basarnos en ellos para hacer una distinción tan fuerte como la que Locke quiere hacer entre hombre y persona?

Dicho en otra forma, Locke puede pretender que su teoría, además de acomodar casos como el de Sócrates dormido y Sócrates despierto, trata de acomodar casos posibles como el del príncipe y el mendigo. Pero resulta despro-

11 Locke parece ignorar el argumento de Aristóteles a ese respecto, según el cual hay que distinguir en la persona la forma o alma de la materia o cuerpo, y lo que confiere identidad o unidad es la continuidad de la misma forma. La tesis de Locke del sustrato o esencia real puede cumplir el papel que Aristóteles asigna a la Forma, pero Locke se resiste a aplicar su teoría de la sustancia a las personas.

12 Es decir, bien puede ser que Locke esté convencido por el PEF de que el ser de las personas debe tener la caracteristica de la certeza y encuentre en los casos contrafácticos un apoyo para esa tesis. Sobre esto volveré más tarde.

${ }_{13}$ Creo posible argumentar, a este respecto, en el sentido de que aun cuando haya un parecido gramatical entre "cambiar una conciencia de una sustancia a otra" y "cambiar o trasplantar un corazón de un cuerpo a otro" este parecido no alcanza a dar inteligibilidad a la tesis de la transmigración de conciencias. 
porcionado que, al intentar acomodar los casos posibles, llegue a conclusiones extremas respecto de casob ordinarios al contar a Sócrates como dos personas: una cuando está despierto y otra cuando está dormido. ${ }^{14}$

Veamos la segunda dificultad de Locke. Dice que "persona" tiene que ver con:

Todo aquello que se funda en una preocupación por la felícidad, el inevitable concomitante de ser consciente; lo que es consciente de placer y dolor, desea que este yo que es consciente sea feliz. $Y$ por lo tanto, cualquier acción pretérita que no pueda reconciliar o apropiar al yo presente mediante la conciencia no podrá importarle, al igual que si nunca la hubiera hecho... (II, XVII, 26.)

Locke piensa, entonces, que la persona se constituye mediante una apropiación de las acciones por medio de la conciencia. La persona es todas aquellas acciones que piensa y concibe como suyas. A Locke le parece que no debe haber castigo o recompensa estando ausente la conciencia de las acciones $\mathrm{u}$ omisiones que se premian o castigan. Asienta que la capacidad de ser sujeto de castigo o recompensa presupone la identidad moral; es decir, si no hubiera identidad moral no habria la capacidad de atribuir castigo o recompensa. Su tesis es que la identidad moral, que es la identidad personal cuando se la considera desde la perspectiva de la personalidad, queda debidamente reconstruida cuando la vemos como una relación mediadora entre varios estados mentales.

Examinaré primeramente las dificultades generales de esta tesis de la identidad moral de las personas, para luego ocuparme de los casos concretos que Locke pretende resolver con su teoría.

En primer lugar, Locke habla de conciencia en el sentido de reflexión o de darse cuenta de los estados mentales que tiene la persona. Piensa en una especie de percepción interna que permite escrutar o percibir los estados mentales. La idea es que alguien ve dentro de sí y se percata de un estado mental actual, o bien, pretérito. En el último caso se trata de la memoria.

En segundo lugar, Locke habla de la conciencia como de una facultad individual, privada, de las personas. Es el ego individual el que se apropia acciones actuales o pretéritas y, con ello, se vuelve responsable de las mismas. Es evidente que la autoatribución individual resulta insuficiente para decidir si se es culpable o no, aun en el propio caso. Tampoco es necesaria, pues es posible que el agente no recuerde el crimen cometido.

\footnotetext{
14 Esta desproporción alimenta la sospecha expresada en la nota 12, a saber, que Locke tiene una preconcepción acerca de la identidad de las personas y sus argumentos buscan justificarla.
} 
En los casos en los que el recuerdo o la conciencia individual resultan insuficientes o no necesarios, el testimonio de los demás resulta supletorio para decidir la cuestión de la atribución de identidad y de responsabilidad, pero aun la afirmación hecha por los demás, aunada a la de la persona moral, puede resultar insuficiente para decidir la cuestión de hecho, a saber, si se es o no culpable, si se tiene o no mérito. Sobre esto volveré al final. Baste subrayar por ahora el deficiente carácter que tiene la teoria de la identidad de Locke en términos de la conciencia o ego individual.

Pasemos a examinar los casos particulares que Locke trae a colación. El primero es un caso de locura parcial, esto es, de intervalos de locura. De éste dice Locke:

Pero si es posible que el mismo hombre tenga diferentes, incomunicables conciencias en diferentes momentos, no hay duda de que el mismo hombre constituirá, en dierentes momentos, diferentes personas... (II, XXVII, 20.)

Locke cuenta dos conciencias, concluye que hay dos personas y argumenta que, puesto que la ley no castiga al enfermo de locura, sino sólo al sensato, debe haber, de acuerdo con la ley, dos personas. Esta es una interpretación muy rara en verdad. ¿Cómo es posible inferir, a partir de la ley o de la práctica legal, una cuestión metafísica? Seguramente puede evitarse la multiplicación de las personas sin tener que ir en contra de la práctica penal. Nada hay, en el caso que menciona Locke, que nos obligue a dejar de pensar en la existencia de una única persona con intervalos de conciencia que evitan la atribución de responsabilidad.

El segundo caso es el de un ebrio y un sonámbulo. Locke dice que ambos son culpables y lo explica así:

Las leyes humanas los castigan a ambos, con una justicia proporcional a su manera de conocimiento - porque en estos casos, ciertamente, no pueden distinguir entre lo real y lo ficticio $y$, por lo tanto, aducir ignorancia en la borrachera o en el sueño no se admite como excusa. (II, XXVII, 22.)

Pace la bárbara equivalencia entre sonambulismo y borrachera, hay que observar aquf, sin embargo, que Locke abandona la conciencia como criterio de atribución de responsabilidad. Por otra parte, especialmente en el caso del sonámbulo, debería decidir - por mor de la congruencia- en forma similar a como decidió el caso del que padece locura a intervalos. En defensa de la incongruente solución que da al caso del borracho, Locke dice: 
Se prueba el hecho en contra de él, pero no se puede probar, en su favor, que le faltó conciencia. (Ibid.)

No se sabe lo que aquí podría demandarse como prueba, más allá de entender propiamente la palabra "borracho". Pero el caso deviene en desastre para la teoría de Locke cuando éste pasa a asegurar:

...en el Gran Día, cuando los secretos de todos los corazones se pongan al descubierto, puede que sea razonable pensar que nadie tendrá que responder de lo que no sabe, pero cada uno recibirá su sentencia final, ya sea que su conciencia lo acuse o lo excuse. (Ibid.)

Esto suena un tanto frívolo, pues ahora resulta que la conciencia ordinaria de una persona no alcanza a constituir su identidad moral y se necesita del auxilio divino para que esa conciencia no sea falsa o simuladora o, en tin, incapaz de alcanzar a decidir lo que la persona es, a saber, culpable o no. Es decir, Locke parece estar introduciendo de nuevo la distinción aparienciarealidad que hace tan atractiva su teoría de la sustancia, sin lograr las ventajas de esta última teoria. ${ }^{15}$ Según esta línea de razonamiento, la identidad de la conciencia no bastaría para asegurar la identidad de una persona; hay algo que es la identidad de la persona y que está más allá de la conciencia que puedẹn tener las personas. Sobre este tema, que ya tocamos antes, habrá que volver aún.

El tercero es un caso de mundos posibles o contrafácticos. Dice Locke:

Pues supóngase que a un hombre se le castigue ahora por lo que hizo en otra vida, de la cual no tiene conciencia alguna. ¿Qué diferencia habría entre concebir este caso como un ejemplo de castigo o concebir que a ese hombre lo crearon miserable? (II, XXVII, 26.)

La conclusión que extrae Locke parece razonable: podemos verlo ya como un castigo, según creían los pitagóricos, o como un caso de miseria humana. Hay que observar, sin embargo, que este ejemplo sólo se suscita sobre la base de concebir que la transmigración de las conciencias a través de varias sustancias es una posibilidad. Creo que sería plausible argumentar -aparte de lo ya dicho acerca de este tipo de casos- que el absurdo de este ejemplo sólo revela el absurdo, todavia más grande, del supuesto metodológico de utilizar casos contrafácticos para decidir la naturaleza de las personas. En todo caso, este

15 Una primera ventaja de la teoría de la sustancia de Locke sería concebir a las personas como seres infradeterminados por nuestros conocimientos actuales y determinables en el futuro: es decir, seres abiertos a la invesitgación, a la observación y al descubrimiento, más allá de nuestra (s) conciencia (s). Otra ventaja fundamental consiste, como se verá después, en que la construcción de la identidad, tal como la concibe Locke, presupone el tener a mano otras identidades de otros continuantes, y el mejor candidato para ocupar este puesto serian las sustancias según las concibe Locke. 
ejemplo solamente requiere incluir la conciencia como una condición necesaria de la identidad personal, nunca como una condición suficiente.

Creo, pues, que Ios casos morales partículares que presenta Locke no ayudan a su tesis de la identidad personal, y que la consideración de los mismos acrecienta la sospecha de que la mismidad de conciencia no es de ninguna manera lo único ni lo más importante que hay acerca de la identidad de las personas.

Finalmente, he de referirme a una tercera fuente de apoyo de la tesis de Locke, la cual, por lo demás, ya ha aparecido en las consideraciones anteriores, a saber, la tesis de la transparencia epistemológica de las personas. Esta es una tesis de tipo general y deriva del principio epistemológico fundamental, o principio de la certeza, que permea toda la filosofía cartesiana.

Locke piensa que las personas son transparentes a sí mismas a través de la conciencia. No hay en ellas una esencia real o sustrato desconocido y una esencia nominal o aparente. Todo lo que es la persona se exhibe a los ojos de la mente o conciencia; la persona se muestra en una autoverificación permanente a toda reflexión de la conciencia. Nada de lo que es la persona escapa a la mirada escrutadora de la conciencia.

Este es un motivo muy general que preside la filosofía del Ensayo; no lo analizaré aquí. Sólo quiero señalar que Locke cree encontrar una relación necesaria entre este motivo epistemológico y las necesidades de la moralidad: es necesario que las personas sean epistemologicamente transparentes, porque, de otra manera, no tendrian sentido las prácticas penales de la sociedad; es decir, el castigo o el premio carecerían de sentido si no supiéramos quiénes somos, esto es, si somos o no la misma persona que llevó a cabo tal o cual acción.

Lo que parece aceptable es que nuestras prácticas penales requieren cierta transparencia epistemológica de la identidad de las personas. no una transparencia total. ${ }^{16} \mathrm{La}$ demanda de una transparencia o certeza total deriva de la aceptación del $P E F$. Ahora bien, no parece haber, en principio, dificultad para conjurar la tesis de una cierta transparencia epistemológica de las personas con una tesis de la identidad personal según la cual la mismidad de una persona es algo más que la conciencia que una persona tiene de sí misma. Dicho en otra forma, no hay una conexión necesaria entre la transparencia epistemológica de las personas y la tesis metafísica de que la identidad de las personas se reduce al enlace de estadios de conciencia.

16 A reserva de que tenga sentido lo que quiere decir una "transparencia total", si la persona apareciera súbitamente, toda ella de golpe, entonces no habría necesidad de construirla. La necesidad solamente surge si la persona ocurre en un devenir, es decir, se muestra en partes sucesivas. La construcción es, para Locke, una empresa que surge por el lado epistemológico, puesto que parte de trozos atómicos de conciencia. Hay, por supuesto, otros motivos para intentar la construcción. 
He considerado las dificultades que surgen de la descripción y comprensión de los casos contrafácticos, así como las que derivan de la atribución de responsabilidad. Finalmente, me he referido a la tesis de la transparencia epistemológica de las personas. De ninguna de estas tres fuentes podemos extraer apoyo para la tesis de Locke de que la identidad personal consiste solamente en la mismidad de una conciencia. ${ }^{17}$

Ahora debo considerar un apoyo más a la teoría de Locke, a saber, su tesis constructivista de la identidad. ${ }^{18}$ Según algunos de los pasajes citados anteriormente, Locke sostiene que la identidad o unidad personal resulta de la acción unificadora de la conciencia, la cual se apropia diferentes existencias y acciones.

En II, XXIII, 23, Locke considera dos casos contrafácticos. Según el primero, habria dos conciencias que habitan una sustancia material o cuerpo; según el segundo, habria una conciencia que habita dos sustancias materiales o cuerpos. La solución de Locke es tajante: en el primer caso cuenta dos personas, en el segundo sólo una.

Supóngase que replicamos venturosamente a esto y logramos demostrar que los casos contrafácticos no pueden resolver la cuestión de cuántas personas hay en cada caso. La cuestión quedaría abierta y Locke podría aún replicar que la forma de contar es arbitraria y convencional y este hecho deja abierta la posibilidad de crear o construir esas identidades. La sugerencia de Locke sería, entonces, que la conciencia/memoria ofrece la mejor posibilidad de llegar a construir la identidad. Sería la mejor porque, entre otras ventajas, tiene la de distinguir las personas de los animales y de los mecanismos o artefactos, sin tener que soportar la pesada carga de una identidad sustancial.

Esta es la razón profunda por la cual la idea de Locke sobrevive a tantos $\mathrm{y}$ tan pesados ataques. No puedo detenerme aquí a examinar esta posibilidad, que tampoco me parece correcta. Sin embargo, para concluir, haré al respecto dos observaciones de distinto tipo. La primera concierne a la convicción -si

17 Hagamos una reflexión sobre el papel que cumple el $P E F$ en los dos últimos motivos que alimentan la tesis de Locke, y que más bien parecen consideraciones justificatorias ofrecidas post facto. La tesis de la atribución de la responsabilidad moral parecía beneficiarse de la tesis de Locke de las personas, pero resultó incapaz de satisfacer el requisito de la certeza. Queda el tercero de los motivos, y éste sí parece responder al PEF. Sin embargo, la conjunción no resulta clara, pues no se trata, como en Descartes, de que las personas sean algo y de que ese algo se manifieste o exprese a la conciencia o ego, sino que la conciencia/ recuerdo - putativa captadora o aprehensora de la persona- es ella misma la que constituye o es la persona. Esto es en verdad difícil de entender.

18 Sobre la teoría constructivista de la identidad en general, y las dificultades que enfrenta, véase el artículo de Sidney Shoemaker "Identity, Properties and Causality", en P. A. French et al. (eds.), Midwest Studies in Philosophy, vol. IV, University of Minnesota, 1979, pp. $321-342$. 
acaso la hay- entre las teorías idealistas de la identidad personal, como la de Locke, y las teorias constructivistas de la identidad. La segunda concierne a la viabilidad de las teorías constructivistas de la identidad, las cuales sostienen que la continuidad de la conciencia/memoria basta para fundar, justificar o explicar la identidad de las personas.

Creo que hay una conexión aparente entre sostener una teorfa idealista de la identidad y sostener una teoría constructivista. Quien sostiene que la identidad personal debe darse en términos de la conciencia o la memoria, rechaza con ello que haya, además, otra cuestión acerca de lo que es la identidad de la persona. Lo que esta tesis dice es que la identidad de una persona es lo que aparece como tal, o que debe darse en términos de lo que aparece y que no hay más que buscar o preguntar. Si la persona tiene conciencia o recuerdo de haber hecho algo, entonces lo hizo, y eso es todo; no hay la pregunta adicional de si real y verdaderamente lo hizo. No hay, por ejemplo, la cuestión: "Y bien, ¿es o no responsable más allá de su conciencia o memoria?"

Por otra parte, las teorías constructivistas de la identidad rechazan que haya un hecho del mundo en el cual consista la identidad, y que tenemos que recoger en nuestro lenguaje. Por el contrario: no hay identidad; somos nosotros los que tenemos que construirla a partir de ciertos requisitos teóricos y prácticos.

En ambos casos vemos operar la misma tendencia, a saber, no hay algo en lo cual consista la identidad de la persona ni la identidad en general: hay lo que aparece y, a partir de ello, tenemos que construir las personas y construir su identidad. En una forma importante, esto significa el rechazo de las cuestiones metafísicas originales: "¿qué son las personas?", "¿qué es la identidad personal?”, "¿qué es ser idéntico?" En este sentido, Locke continúa el camino iniciado en su teoría de la sustancia, es decir, dejar de lado las consideraciones o preguntas de la tradición metafísica. En el caso de la sustancia, esto equivale a dejar el apriorismo y convertir la cuestión de la sustancia en una cuestión empírica. En el caso de la identidad personal, ese "dejar de lado" equivale a algo más radical: a subvertir la cuestión misma, decidiendo que es una cuestión mal formada y que pregunta por algo ininteligible. En lugar de esa demanda imposible hay que formular otras: "¿cómo se presentan las personas a sí mismas?”, "¿cómo se construye la persona a partir de esas presentaciones?", "¿cómo se construye la identidad de los objetos o entes?"

Empero, la conexión es sólo aparente, pues no hay razón para condenar una teoría constructivista de la identidad que no recurre a la conciencia/ memoria o a otro predicado mental, sino más bien a algo corporal o material. ${ }^{19}$

19 En Locke se conjuntan el idealismo mentalista de la teoría de las personas (las per:6onas son su conciencia) con el constructivismo (la identidad de las personas se forma $o$ 
Respecto a la segunda observación, debo distinguir dos cuestiones. Por un lado, la relativa a la construcción de la identidad de las personas. Por el otro, la cuestión, más general, acerca de la construcción de la identidad en general. Esta segunda cuestión no la consideraré aquí.

Locke se conforma con asegurarnos que la conciencia es la clave para resolver la cuestión acerca de los continuantes que son las personas, pues solamente ella es capaz de enlazar diferentes existencias y acciones a manera de formar una unidad. Sin embargo, según suelen hacer los filósofos, se calla el cómo, esto es, no nos dice cómo la conciencia/memoria cumple ese papel de enlace, el tipo de relación que es ni, sobre todo, cómo se basta a si misma para construir la historia de un tal continuante.20

La idea que se deriva de Locke es que debe haber una relación $R$ entre conciencias o estado de conciencia/recuerdo, que permita enlazar las diferentes conciencias. Pero, como la conciencia es atómica y tiene una existencia precaria e intermitente, debe haber algo que permanece o continúa a través de la transmigración de las conciencias, pues de otra manera habría que suponer otra conciencia que permanece o continúa mientras la primera desaparece. Es decir, solamente recurriendo a otro continuante que permanece y permite trazar el encadenamiento de conciencias podría evitarse la arbitrariedad que amenaza a la tesis constructivista. ${ }^{22}$

Pero, entonces, eso quiere decir que la tesis constructivista no es última, y requiere o supone la identidad de otro continuante. Bueno, esta idea es precisamente la que apareció antes en varios lugares, a saber, al hablar de la insuficiencia de la teoría de Locke para resolver los casos de responsabilidad y al tratar su tesis de la transparencia de la conciencia. Una forma sucinta y alternativa de asentar esto es decir que la conciencia solamente constituye una condición necesaria de la identidad personal, esto es, sólo alcanza a constituir un tipo de condición necesaria, pero sólo puede alcanzar esto, si se toman en cuenta otras condiciones igualmente necesarias y quizá suficientes. Por lo tan-

hace a partir de átomos de conciencia), pero la tesis constructivista de la identidad es inde. pendiente de la tesis ontológica de que las personas son conciencia.

Deseo mencionar aqui que la tesis constructivista de la identidad de Locke absorbe el problema de la identidad a través del tiempo en el problema de la unidad, porque la unidad de un continuante se va constituyendo en cada instante a través del tiempo.

$20 \mathrm{Si}$ bien Locke guarda silencio sobre esto, el problema ha sido recogido por los neolockeanos, quienes han tratado de ofrecer una teoría a este respecto a partir de la adición de Hume, de acuerdo con la cual hay que introducir además un elemento causal. $C f$. por ejemplo H. P. Grice, "Personal Identity", en J. Perry (ed.), Personal Identity, University of California Press, 1975. Véase mi nota bibliográfica sobre este libro en Critica, vol. IX, núm. 27, 1977. También es importante la forma en que Sidney Shoemaker intenta contsruir una relación que salve la tesis de Locke, vis à vis un mundo de casos contrafácticos, en Las personas y su pasado. Cuadernos de Critica, núm. 8, 1981.

21 Sobre esta y otras dificultades de la tesis de Locke puede consultarse la antologia de A. Rorty The Identities of Persons, University of California Press, 1976. (Una reseña de este libro apareció en Crítica, vol. XII, núm. 36, 1980.) 
to, en ningún caso podemos dar una lectura cartesiana o, lo que es equivalente, una lectura idealista y mentalista de la teoría de Locke, si queremos rescatar su aportación dentro de una teoría adecuada de la identidad de las personas. $^{22}$

Si lo anterior es correcto, se abre entonces la doble posibilidad de complementar la teoría de Locke con otras condiciones necesarias de la identidad personal y, a la vez, de incorporar la noción de sustancia en la elucidación de la identidad de las personas. Esta complementación equivale a negar la tesis de Locke de que las personas no son sustancias y concomitantemente equivale a afirmar que la tesis de la sustancia del propio Locke debe aplicarse también a las personas. ${ }^{23}$ Esta es, en parte, la tesis de Leibniz. ${ }^{24}$

ENRIQUe VILlanueva

Instituto de Investigaciones Filosóficas

Universidad Nacional Autónoma de México

22 Esta es la conclusión que saca Shoemaker después de hacer una ardua defensa de la tesis de Locke en Las personas y su pasado. Shoemaker salva la tesis de Locke del cargo de circularidad introduciendo la noción de los cuasi-recuerdos, que están enlazados causalmente; pero el enlace causal requiere de la existencia de otro continuante material, que resulta ser el cerebro.

23 Un defensor de la teoria de la sustancia para el caso de la identidad personal completará la refutación de las teorías construccionalistas de la identidad argumentando que los casos de memoria, así como un buen número de casos contrafácticos y los casos sociales e históricos, pueden acomodarse sin mayor dificultad con la explicación sustancial de la identidad. Esta es la tarea que Leibniz desarrolla en parte cuando responde a Locke en los Noveaux Essais sur le Entendement Humain.

24 Deseo agradecer al maestro José Antonio Robles sus sugerencias tanto respecto de las traducciones del texto de Locke como del resto del trabajo. Por supuesto, ninguno de los defectos que aún padece el presente trabajo debe atribuirsele. 